\title{
Prevalence of Workplace Violence Against Nurses Working in Hospitals: A Literature Review
}

\author{
Amani Mobaraki ${ }^{1,2, ~}{ }^{*}$, Rawan Aladah ${ }^{1}$, Rehaf Alahmadi ${ }^{1}$, Taghreed Almuzini ${ }^{1}$, Loujain Sharif ${ }^{1}$ \\ ${ }^{1}$ Faculty of Nursing, King Abdulaziz University, Jeddah City, Saudi Arabia \\ ${ }^{2}$ Faculty of Nursing, Jazan University, Jazan City, Saudi Arabia
}

Email address:

amanimobarki@hotmail.com (A. Mobarki)

${ }^{*}$ Corresponding author

\section{To cite this article:}

Amani Mobaraki, Rawan Aladah, Rehaf Alahmadi, Taghreed Almuzini, Loujain Sharif. Prevalence of Workplace Violence Against Nurses Working in Hospitals: A Literature Review. American Journal of Nursing Science. Vol. 9, No. 2, 2020, pp. 84-90.

doi: 10.11648/j.ajns.20200902.19

Received: February 5, 2020; Accepted: March 11, 2020; Published: April 1, 2020

\begin{abstract}
The rise in documented rates of workplace violence (WPV) in healthcare facilities has become a major issue for healthcare providers worldwide, particularly nurses. The exposure to such violence can result in grave consequences for the physiological and psychological well-being of nurses. However, previous studies in the field rarely differentiated between the types of workplace violence, their sources, and their impact on nursing staff. Aim: This review aims to describe the prevalence of WPV against nurses in hospitals. Methods: A literature review, based on the PRISMA guidelines, of studies of qualitative, quantitative, and mixed method design that focus on nursing WPV. A literature search is conducted using CINAHL, MEDLINE, and PubMed databases, via the Saudi Digital library. The search includes studies published during the last five years. All of the studies included are critically appraised using the critical appraisal tool developed by Hawker et al. (2002). In total, 25 studies matching the inclusion criteria emerge from the selection process, and are included in the review. Results: A total of four themes emerge from the literature related specifically to WPV towards nurses, namely common settings where violence is experienced, common types of violence, the characteristics of perpetrators, and the overall impact of violence on nurses. The most common hospital settings where WPV is experienced include the emergency and psychiatric departments, and the Neonatal Intensive Care Unit (NICU). The most prevalent forms of WPV are, in order of prevalence from most common to least, verbal, physical, psychological, and horizontal violence. Rates of WPV exposure are found to vary according to world region, with the highest rates for physical violence, sexual harassment, and horizontal abuse in the United States (US), and the highest rates of psychological violence and bullying in Asia. Patients, and patients' families/friends are the most common perpetrators of WPV. The consequences of WPV are found to include a diminished physical and emotional well-being, linked to an increase in job dissatisfaction, burnout, absenteeism, and resignation from work. Conclusion: This review recommends that more high-quality research should be conducted in this field, using systematic techniques and standardized tools that enable generalization across studies. Furthermore, it recommends that preventive action in the form of multi-level educational programmes, and legislative polices to support and protect the rights of those reporting WPV, are urgently required.
\end{abstract}

Keywords: Workplace Violence, Bullying, Psychiatric Hospitals, Psychiatric Nurses

\section{Introduction}

Violence against nursing staff in all its forms, including sexual, verbal, and behavioral, is widespread in all societies worldwide, and especially in hospitals. The current methods employed to reduce workplace violence (WPV) towards nurses varies in different regions. The hospital environment in which nurses work can affect the quality of care they provide to their patients, and can sometimes result in nursing errors.

Previous studies indicated that among the most common perpetrators of WPV towards nurses are patients and their relatives, as well as colleagues in the workplace, such as supervisors, and those who have authority over nurses [19, 29]. Such violence affects nurses in many ways, for example 
causing disruption to their work, and undermining their overall satisfaction with providing nursing care [4]. Moreover, some studies found that WPV can cause certain psychological conditions for nurses who are exposed to violence. The definition of violence is the use of physical force that causes injury, damage, abuse, or destruction [19].

There is a higher risk of nurses encountering WPV than other professions, both within and outside the hospital setting [8]. A possible explanation for this greater risk is that the threat is always obfuscated, and is rarely discussed openly. In order to improve conditions within healthcare, it is important to determine the frequency and risk of such violence in hospitals.

Nurses are negatively affected by the violence they encounter in their daily work, as it has serious effects on their mental and physical health, as well as on their job satisfaction, productivity, and anxiety levels. This causes feelings of anger and powerlessness, which affect the quality of their patient care, and in severe cases can drive nurses to leave their positions [16]. These harmful consequences have prompted an urgent need for research into the causes of WPV against nurses, and a need to develop evidenced-based policies to provide safer working environments for them [13].

This review examines the findings of previous studies concerning WPV in hospitals, published during the last five years, that focus on the types of such violence, and their impact on nurses. It provides an in-depth analysis of the effects of violence on nurses' work productivity and psychological status. All of the studies that matched this study's criteria, regardless of their methodology, whether qualitative, quantitative, or mixed methods, were critically appraised, in order to provide a wide representation of research evidence, and to describe the prevalence of WPV against nurses in hospitals.

\section{Search Strategy}

A literature review was conducted using a number of different databases, including CINAHL, MEDLINE, and PubMed, via the Saudi Digital library database, together with an internet search, using Google Scholar. The electronic search of the CINAHL, Medline, and PubMed databases was completed on 20 November 2018, and produced a total of 67 records. The search terms included 'violence' and 'nurses'. Search limits were applied, including year of publication, which was 2013 to the date the search was conducted, and studies written in the English language. Once the duplicate articles were removed, the remaining 58 articles were screened by title, which produced 36 potentially relevant articles, according to the abstracts, and the full-text electronic copy availability.

The research team then reviewed each of the 36 articles using the inclusion criteria, namely articles whose sample was nurses, including nursing aides, nursing students, and intern nurses of both genders, and of all ages. The studies were also required to have been conducted in hospitals, in any unit, with a focus on WPV against nurses of any kind. Articles were excluded if their primary focus was not WPV; if their setting was community centres or homecare settings; if the sample included other healthcare workers, with no specific reporting of the rate of the nurses' presence; or if they were concerned with domestic violence. The final number of articles included in the review was 25, 10 of which were retrieved from CINHAL, nine from Medline, and six from PubMed (see Figure 1 for details of the study's selection process).

\section{Results}

The results are presented in the form of a description of the characteristics of the studies included, followed by a critical appraisal of the studies, and finally an evaluation of the themes extracted. A broad range of studies regarding different aspects of WPV directed at nurses was identified as part of the literature search. Each study included was reviewed individually by comparing each paper's aims and/or objectives, the study design, the method of data collection and analysis, the main findings, and the implications for practice. In order to identify the research themes, a review matrix was employed to organize the key studies by theme. This process helped to highlight the common themes that emerged across the studies, and to identify the studies' shared elements, together with their differences.

The data was extracted from 26 studies, the research design of which were: qualitative $(n=5)$, quantitative $(n=20)$, and mixed methods $(n=1)$. The data extraction table contained a description of several elements that were essential for identifying the unique and pertinent data from each of the articles involved, namely the studies' reference; data base; region; design; sample number; type of participants; type of harassment, and its impact; tool/outcome measure; key findings; and limitations. Of the 25 studies reviewed, the majority were conducted in Asia $(n=9)$, followed by North America $(n=7)$, with a minority published in Africa $(n=2)$ and the Middle East $(n=2)$.

The design of the studies included was as follows: cross-sectional studies $(\mathrm{n}=13)$, longitudinal studies $(\mathrm{n}=1)$, and descriptive surveys $(n=11)$ that included quantitative methods, or qualitative methods using semi-structured interviews $(n=4)$. The sample population of 11 of the articles was relatively large, with a total of 300 or more nurses, while 14 articles had samples of fewer than 300 nurses. The specific location of the act/s of violence also varied, but the majority occurred in psychiatric units $(n=5)$, followed by in-patient units.

In terms of the type of violence involved, 14 articles concentrated on verbal violence, while 17 studies discussed physical violence. Meanwhile, six studies focused on psychological abuse, and two mentioned emotional abuse. The studies also examined the nature of the perpetrators of the violence, and the impact of the violence on the nurses concerned. With regards to the perpetrators, the studies often included patients and co-workers among the most prevalent perpetrators of WPV. In total, 17 studies mentioned patients as the primary perpetrator, while 14 cited the patients' family members as the perpetrators. Nine of the studies suggested that co-workers and staff members can also be the perpetrators of WPV. 
Meanwhile, the studies reported that the impact of the violence on the victim, namely the nurses, and their relationship with the perpetrators, included frustration, physical pain, emotional distress, feelings of anger and powerlessness, high anxiety levels, and other mental health problems.

Finally, the tools employed by the studies took the form of survey questionnaires $(\mathrm{n}=17)$, including those developed and modified by the authors of the study $(n=8)$; interviews $(n=3)$; and three employed mixed methods, combining questionnaire with interviews.

Critical appraisal is the process of examining research trustworthiness, and its value and relevance in a particular context. It also enables researchers to eliminate irrelevant or weak studies, to identify the most relevant papers, and to assess the validity of studies [26]. For the purpose of the critical appraisal process of this review, the authors employed the critical appraisal tool developed by Hawker et al. (2002) to systematically appraise the quality of all of the studies included. This appraisal tool includes nine items for examining the abstract, reported method, sampling, data analysis, ethics and bias, the generalizability of the findings of the study, and whether they are transferable. The quality of studies was measured using a 4-point Likert scale: (1) Very poor, (2) Poor, (3) Fair, and (4) Good.

Of the 25 studies included, 11 articles were deemed to be of fair quality, while 15 were considered to be of good quality. The majority of the studies rated 'Fair' had some description of their context and setting, but required more information to replicate the study, or to compare it with others. In addition, the ethical issues, such as confidentiality, sensitivity, and bias were not addressed by these studies. Meanwhile, the studies rated 'Good' included a well-structured abstract that provided complete information and clear titles. Moreover, their findings were easy to understand, and were in presented in a logical progression. The tables, if any were present, were explained in the text, and the results related directly to the aim and objectives of the study.

A total of four themes emerged from the literature related specifically to WPV towards nurses, namely common settings where violence is experienced, types of violence, characteristics of the perpetrators, and the impact of violence on nurses. These themes are examined in detail in the next sub-sections of this review.

\subsection{Common Settings of WPV}

The incidence rates of WPV in the studies in this review encompassed different locations, including county-level hospitals, general hospitals, tertiary hospitals, teaching hospitals, and both rural and urban primary care facilities. The studies focused on common settings in these hospitals, such as the emergency and psychiatric departments, the Neonatal Intensive Care Unit (NICU), labor and delivery units, teaching hospitals' surgical units, Intensive Care Units (ICU), geriatric wards, and general hospital settings [5, 6, 12, 28, 34]. The majority of the studies were conducted in general hospitals $(n=10)$, followed by psychiatric units $(n=5)$, while the remainder were conducted in NICU and labor units $(n=2)$, emergency departments $(n=2)$, surgical units $(n=3)$, tertiary hospitals $(n=2)$, teaching hospitals $(n=2)$, and ICU and pediatric departments $(n=1)$. The majority of the studies found that WPV is most prevalent in psychiatric units, as a result of dealing with patients suffering from agitation, violent behavior, withdrawal symptoms, and suicidal attempts [29].

\subsection{Types of Violence}

Nurses are exposed to different types of WPV. The majority of the studies agreed that the most common types of violence they encounter in hospitals are verbal $(n=7)$, physical $(n=4)$, horizontal $(n=3)$, and laterally violent behaviors $(n=1)[1,3,19,21,23]$.

Nurses working in various types of hospital/units are at high risk of experiencing physical violence at work, which is the second most common type of violence, after verbal violence. The forms that physical violence can take include being confronted and chased; being hit; kicked; grabbed, or punched; spat at; strangled; and the use of a weapon, or the use of the environment, such as breaking a window, in an act of physical or verbal violence [25]. More than half of the studies focused on physical violence $(\mathrm{n}=15)$. According to Binil, Sudhaker, \& Hegde (2016), 58\% of the nurses in their study had experienced physical assault perpetrated by patients during their shifts, on more than five occasions. Most nurses tended to be hit while restraining or secluding patients in psychiatric units [6].

Meanwhile, horizontal WPV and bullying has been reported and documented widely among healthcare professionals, and causes many negative outcomes for nurses, patients, and healthcare workers [28]. The terms horizontal violence, lateral violence, and horizontal hostility all describe hostile, aggressive, and harmful behavior by a nurse, or a group of nurses, towards a co-worker or group of nurses by their actions, attitudes, verbal or other behavior, or generally between individuals at the same power level [28]. Examples of horizontally violent behavior include the raising of eyebrows and face-making; actions such as turning away, or ignoring; information withholding; planned disruption, such as intentionally establishing a negative situation; infighting with peers; scapegoating individuals; betrayal; failing to respect the privacy of a co-worker; and verbal aggression, such as making sarcastic comments, or name-calling. Hospital units, such as antepartum, the NICU, and the nursery unit, had the lowest incidence rates of such violence.

According to the study conducted by Armmer \& Ball (2015), there is a positive relationship between the experience of horizontal violence, and the intention to resign from a job. Moreover, horizontal violence can be problem even when Registered Nurse's (RNs) have received formal education on the matter, and if left untreated, it is likely to have an impact on nursing attrition rates.

Meanwhile, lateral violence takes common forms, such as "non-verbal insinuation, verbal offenses, declining and underrating one's activities, withholding information, interruption, infighting, blaming, betraying, not respecting privacy of the healthcare professionals and confidence breaking" [4]. The only study included in this review that 
addressed lateral violence was conducted by Dahlby \& Herrick (2014), using a one-time pre-test, post-test evaluation study design, with a total of 26 nurses. The data was collected using the Lateral and Vertical Violence in Nursing Survey, developed by Stanley et al. (2007) to measure the perceived incidence and severity of lateral violence. The study's participants were able to identify the causes of lateral violence in the work-place better after attending an educational intervention on decreasing lateral violence in the workplace through awareness and cognitive rehearsal. Moreover, the suggestions made by the educational intervention had a positive effect, creating a healthier working environment, and decreasing the incidence of laterally violent behaviors. However, due to the small sample size of the study, further evaluation is required to determine the effectiveness of such an intervention over time.

\subsection{Characteristics of the Perpetrators and Victims of WPV}

All of the studies included in this review reported that the perpetrators of WPV were either patients, relatives, doctors, managers, or co-workers. The studies indicated that patients and relatives were more likely to be the perpetrators than co-workers [32], and consequently suggested that support for nurses who experience physical and verbal WPV must be made available, and that nurses should be encouraged to report and confront such violence. Moreover, healthcare managers must heed nurses' reports of violence. Many studies conducted with healthcare workers of both genders found that more female victims $(86 \%)$ reported physical violence, and more male victims $(81 \%)$ reported verbal violence [2].

\subsection{Impact of Violence}

The consequences of direct violence, such as physical abuse, were investigated by two of the studies included in this review, six of the studies addressed the consequences of indirect violence in the form of emotional abuse, and others addressed the consequences of both forms of WPV.

The studies that addressed physical WPV reported that it can cause immediate consequences, such as injuries, including bites, bruises, lacerations, and hair loss [23, 32]. Meanwhile, the studies concerning indirect violence reported that it can cause emotional reactions, such as anger, fear, high levels of stress, anxiety, exhaustion, frustration, feelings of vulnerability, and feelings of being violated psychologically. According to these studies, nursing staff possess the professional skills and abilities required to employ different kinds of interventions to over-come and prevent violent situations. An important finding was that higher levels of anxiety caused by WPV are associated with an increased risk of violence.

Moreover, WPV causes nurses to leave their positions, and to experience job dissatisfaction, and a desire for a workplace change, and it also has a huge impact on the nurse-patient relationship, ultimately engendering a lower quality of healthcare $[22,27]$.

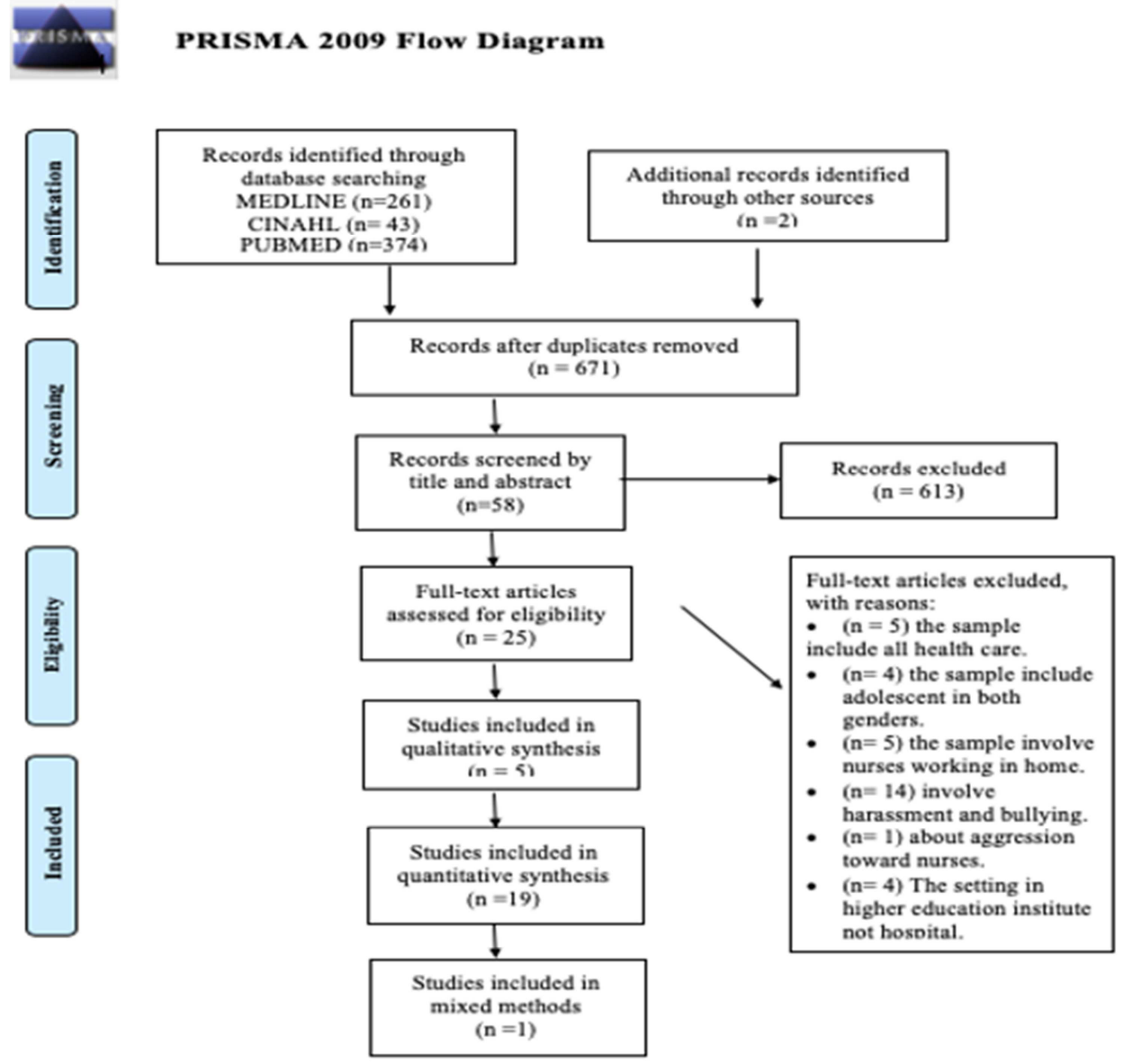

Figure 1. Flow diagram of the approach employed by this study, based on PRISMA guidelines (Moher et al., 2009). 


\section{Discussion}

This systematic review confirmed the presence of WPV against nurses in diverse settings, in different countries, and presented the findings under four themes: the common settings where such violence occurs, the types of violence, the characteristics of the perpetrators and victims of violence, and the impact of violence. The analysis of the studies included in this review determined that the most common setting and type of violence is physical and verbal violence that is most prevalent in psychiatric units and emergency departments. Horizontal violence was found to be the dominant form of violence in labor and delivery units, compared with the other units. Although there were differences in the incidence rates of sexual violence, relatively few studies addressed this type of WPV.

It was found that the WPV encountered by nurses can be under-reported, at least officially, and that these under-reporting tendencies vary across different regions of the world, due to cultural sensitivity to the exposure of violence, especially sexual harassment. However, some studies found that managers and staff at a similar power level reported the incidences of WPV more than staff nurses or student nurses. Furthermore, nurses aged 40-44 years reported sexual harassment more frequently than staff nurses aged 30 years and under [11, 12].

The findings of this review confirmed that the reasons why nursing staff become victims of WPV is most often related to gender, atmosphere, environment, and the characteristics of the nursing staff members [5]. In addition, poor communication skills, and inappropriate professional communication, were sometimes cited by the studies' participants as reasons for workplace violence [5]. The review also highlighted the importance of communication skills when managing violent situations, for example in psychiatric or emergency units.

This review found that the impact of WPV can include increased emotional stress that prompts nursing staff to leave their position in extreme cases, and also creates job dissatisfaction, exhaustion, and frustration, that can cause a decrease the quality of care provided by the victims to their patients. Moreover it was found that exposure to violence can cause burnout and high turnover rates among nurses, and the experience of multiple forms of violence is related to psychological trauma. According to the study conducted by Stevenson et al. (2015), nurses' fear of their own vulnerability continues for a long time after being exposed to WPV, and they report feelings of uncertainty about their safety at work.

An assessment of the physical and verbal violence discussed in the studies included in this review found that females are at a higher risk of experiencing verbal WPV than male, while males are at higher risk of experiencing physical WPV [29]. There were also differences in the rate of exposure to WPV according to world region, with each region possessing a distinctive pattern. For example, psychological violence and bullying were most frequent in Asia, while the
United States (US) ranked the second highest in terms of rates of physical violence, sexual harassment, and horizontal abuse. Meanwhile, Africa ranked the lowest for non-physical violence, and Europe ranked the second lowest for physical violence, bullying, and sexual harassment. It therefore appeared that nurses in the US had the highest overall exposure to physical WPV, while nurses in Asia had the least. Meanwhile, nurses in Africa and Europe experienced relatively high levels of nonphysical violence, and relatively low levels of physical WPV. In part, this low rate of physical WPV might be due to the fact that male nurses reported the most experiences of physical WPV. This review did not address the cultural differences regarding the experience of violence in the nursing profession.

It is necessary to heed all types of violence in different nursing settings, and to highlight the importance of introducing interventions for management and staff to address the matter. New protocols should be developed to facilitate the reporting process of incidences of WPV, and to ensure healthcare workers' safety in their workplace, in all situations.

There are two previous systematic reviews of violence against nurses in different settings, the first of which was conducted by Spector et al. (2014), and found that the rates of physical violence and sexual harassment were highest in the Anglo region, while the rates of nonphysical violence and bullying were highest in Middle East region. The study found that the rates of bullying and sexual harassment were the lowest in Europe. In addition, the study reported that instances of violence instigated by patients or relatives were the highest in France, and the lowest in The Netherlands. According to the authors, patients were the most common perpetrators of violence in the Anglo and European regions, while in Asia and Middle East, the perpetrators were usually the patient's family members or friends, a difference that Spector et al. (2014) speculated may be due to the cultural background, values, and sensitivities.

The second extant systematic review was conducted by Konttila et al. (2018), and addressed the reasons for, forms of, and consequences of violence in psychiatric settings. It reported that the causes of violence often concern the patients' influence on the nursing staff, and found that in psychiatric settings, verbal violence is the most common type of violence, and it most frequently causes the nursing staff concerned to experience psychological repercussions. The study's findings highlighted the importance of staff interventions for managing all kinds of violent situations.

\section{Limitations}

The major limitation of this literature review was the paucity of previous studies in the field, since only 21 articles exclusively featured nurses. In addition, some of the studies neglected to consider the matter of the under-reporting of the issue.

A second limitation was the heterogeneity of the studies, which meant that they were not all comparable across type, 
setting, source, and region. This was a limitation in terms of the degree of confusion among the different studies' characteristics, as well as the ability to compare across world regions, which was also affected by the limited number of extant studies. Unfortunately, few of the studies employed the same measures and procedures that would enable in-depth comparisons across regions.

The third limitation was that there was no standardization in terms of the measurements or the methods employed by the studies, which caused problems with clarifying and distinguishing the types of violence involved. The studies varied in terms of the specific questions they asked about violence, with some separating physical from non-physical violence, such as Li et al. (2018), and others combining the two, such as Ridenour et al. (2015). This variation of measurement caused a disparity in the incidences of violence reported by the nurses in each study. Moreover, some of the studies had a small sample size, such as Reynolds et al. (2014) and Stevenson et al. (2015), that limited the comparisons that could be made to achieve accurate results.

\section{Implications for Research and Nursing Practice}

This review provided an assessment of WPV exposure rates in nursing. Although the high rate of physical WPV is well known, and has been the focus of workplace interventions in many healthcare organizations, there is less recognition of other types of violence, such as bullying and sexual harassment. While the majority of physical violence towards nursing staff is committed by patients, violence prevention programmes must be inclusive and comprehensive, addressing the matter in relation to patients, their families and friends, and staff members, including both nurses and physicians.

Only by addressing all types and sources of violence can nurses' workplaces become safer environments. Moreover, although the exposure to WPV is widespread, there are regional and country differences in its incidence rates and sources. Hence, interventions should be customized to the particular issues of WPV experienced in a specific setting. It is also important to determine whether the low rates of sexual harassment in some regions are due to an actual low incidence rate, or to its under-reporting, as this would assist in recognizing the extent to which efforts must be directed toward sexual harassment prevention in these regions.

\section{Conclusion}

This literature review highlighted the problem of the various types of WPV experienced by nurses on a daily basis in the different regions of the world. According to the findings of the review, verbally violent behavior is the main form of WPV reported by nurses worldwide, followed by physically violent behavior that has dangerous consequences, such as injuries, and finally other types of violence. Daily exposure to WPV can affect nurses' mental and physical well-being, which can impact on the quality of care they provide to their patients. According to the results of this literature review, there is lack of policies and assertive legislation in most of the nursing settings where WPV occurs, which causes a higher and more frequent risk of such violence occurring. There is therefore a need for further research to determine solutions for addressing this problem, such as reporting process policies, educational sessions, and suitable interventions to confront such violent behavior in its various forms.

\section{References}

[1] Aivazi, A. A., Menati, W., Tavan, H., Navkhasi, S., \& Mehrdadi, A. (2017). Patients' bill of rights and effective factors of workplace violence against female nurses on duty at Ilam teaching hospitals. Journal of injury and violence research, 9 (1), 1. (Aivazi et al., 2017)

[2] Abed, M., Morris, E., \& Sobers-Grannum, N. (2016). Workplace violence against medical staff in healthcare facilities in Barbados. Occupational medicine, 66 (7), 580-583.

[3] Aksakal, F. N. B., Karaşahin, E. F., Dikmen, A. U., Avci, E., \& Özkan, S. (2015). Workplace physical violence, verbal violence, and mobbing experiencedby nurses at a university hospital. Turkish journal of medical sciences, 45 (6), 1360-1368.

[4] Armmer, F., \& Ball, C. (2015). Perceptions of horizontal violence in staff nurses and intent to leave. Work, 51 (1), 91-97.

[5] Avander, K., Heikki, A., Bjerså, K., \& Engström, M. (2016). Trauma nurses' experience of workplace violence and threats: short-and long-term consequences in a Swedish setting. Journal of trauma nursing, 23 (2), 51-57.

[6] Binil, V., Sudhaker, C., \& Hegde, S. (2016). Care Providers Experience of Violence in Psychiatric Setting. International Journal of Nursing Education, 8 (4), 217-220.

[7] Boyle, M., Koritsas, S., Coles, J., \& Stanley, J. (2007). A pilot study of workplace violence towards paramedics. Emergency Medicine Journal, 24 (11), 760-763.

[8] Camerino, D., Estryn-Behar, M., Conway, P. M., van Der, B. I. J. M., \& Hasselhorn, H.-M. (2008). Work-related factors and violence among nursing staff in the European NEXT study: a longitudinal cohort study. International journal of nursing studies, 45 (1), 35-50.

[9] Chang, H. E., \& Cho, S.-H. (2016). Workplace violence and job outcomes of newly licensed nurses. Asian nursing research, 10 (4), 271-276.

[10] Cheung, T., Lee, P. H., \& Yip, P. S. (2017). Workplace violence toward physicians and nurses: prevalence and correlates in Macau. International journal of environmental research and public health, 14 (8), 879.

[11] Cheung, T., \& Yip, P. S. (2017). Workplace violence towards nurses in Hong Kong: prevalence and correlates. BMC public health, 17 (1), 196.

[12] Cho, O.-H., Cha, K.-S., \& Yoo, Y.-S. (2015). Awareness and attitudes towards violence and abuse among emergency nurses. Asian nursing research, 9 (3), 213-218. 
[13] Cowman, S., \& Bowers, L. (2009). Safety and security in acute admission psychiatric wards in Ireland and London: a comparative study. Journal of clinical nursing, 18 (9), 1346-1353.

[14] Dahlby, M. A., \& Herrick, L. M. (2014). Evaluating an educational intervention on lateral violence. The Journal of Continuing Education in Nursing, 45 (8), 344-350.

[15] Fafliora, E., Bampalis, V. G., Zarlas, G., Sturaitis, P., Lianas, D., \& Mantzouranis, G. (2016). Workplace violence against nurses in three different Greek healthcare settings. Work, 53 (3), 551-560.

[16] Gates, D. M., Gillespie, G. L., \& Succop, P. (2011). Violence against nurses and its impact on stress and productivity. Nurs Econ, 29 (2), 59-66.

[17] Hawker, S., Payne, S., Kerr, C., Hardey, M., \& Powell, J. (2002). Appraising the evidence: reviewing disparate data systematically. Qualitative health research, 12 (9), 1284-1299.

[18] Jiao, M., Ning, N., Li, Y., Gao, L., Cui, Y., Sun, H.,... Hao, Y. (2015). Workplace violence against nurses in Chinese hospitals: a cross-sectional survey. BMJ open, 5 (3), e006719.

[19] Koukia, E., Mangoulia, P., Papageorgiou, D. E., Gonis, N., \& Katostaras, T. (2014). Violence against health care staff by peers and managers in a general hospital in Greece: a questionnaire-based study. Australian Journal of Advanced Nursing, The, 31 (4), 45.

[20] Konttila, J., Pesonen, H. M., \& Kyngäs, H. (2018). Violence committed against nursing staff by patients in psychiatric outpatient settings. International journal of mental health nursing.

[21] Kvas, A., \& Seljak, J. (2015). Sources of workplace violence against nurses. Work, 52 (1), 177-184.

[22] Li, P., Xing, K., Qiao, H., Fang, H., Ma, H., Jiao, M.,... Gao, L. (2018). Psychological violence against general practitioners and nurses in Chinese township hospitals: incidence and implications. Health and quality of life outcomes, $16(1), 117$.

[23] Mantzouranis, G., Fafliora, E., Bampalis, V. G., \& Christopoulou, I. (2015). Assessment and analysis of workplace violence in a Greek tertiary hospital. Archives of environmental \& occupational health, 70 (5), 256-264.

[24] Moher, D., Liberati, A., Tetzlaff, J. and Altman, D. G. (2009). Preferred reporting items for systematic reviews and meta-analyses: the PRISMA statement. Annals of internal medicine, 151, 264-269.

[25] Nguluwe, B., Havenga, Y., \& Sengane, M. (2014). Violence experienced by nurses working in acute care psychiatric wards at a Gauteng Hospital. Africa Journal of Nursing and Midwifery, 16 (1), 60-74.
[26] Parkes, J., Hyde, C., Deeks, J. J., \& Milne, R. (2001). Teaching critical appraisal skills in health care settings. Cochrane Database of Systematic Reviews (3).

[27] Purpora, C., Blegen, M. A., \& Stotts, N. A. (2015). Hospital staff registered nurses' perception of horizontal violence, peer relationships, and the quality and safety of patient care. Work, $51(1), 29-37$.

[28] Reynolds, G., Kelly, S., \& Singh-Carlson, S. (2014). Horizontal hostility and verbal violence between nurses in the perinatal arena of health care. Nursing Management (2014), 20 (9), 24.

[29] Ridenour, M., Lanza, M., Hendricks, S., Hartley, D., Rierdan, J., Zeiss, R., \& Amandus, H. (2015). Incidence and risk factors of workplace violence on psychiatric staff. Work, 51 (1), 19-28.

[30] Schablon, A., Wendeler, D., Kozak, A., Nienhaus, A., \& Steinke, S. (2018). Prevalence and Consequences of Aggression and Violence towards Nursing and Care Staff in Germany-A Survey. International journal of environmental research and public health, 15 (6), 1274.

[31] Shi, L., Zhang, D., Zhou, C., Yang, L., Sun, T., Hao, T.,... Mu, Y. (2017). A cross-sectional study on the prevalence and associated risk factors for workplace violence against Chinese nurses. BMJ open, 7 (6), e013105.

[32] Spector, P. E., Zhou, Z. E., \& Che, X. X. (2014). Nurse exposure to physical and nonphysical violence, bullying, and sexual harassment: A quantitative review. International journal of nursing studies, 51 (1), 72-84.

[33] Stanley, K. M., Martin, M. M., Nemeth, L. S., Michel, Y., \& Welton, J. M. (2007). Examining lateral violence in the nursing workforce. Issues in Mental Health Nursing, 28 (11), $1247-1265$

[34] Stevenson, K. N., Jack, S. M., O’Mara, L., \& LeGris, J. (2015). Registered nurses' experiences of patient violence on acute care psychiatric inpatient units: an interpretive descriptive study. BMC nursing, 14 (1), 35.

[35] Tiruneh, B. T., Bifftu, B. B., Tumebo, A. A., Kelkay, M. M., Anlay, D. Z., \& Dachew, B. A. (2016). Prevalence of workplace violence in Northwest Ethiopia: a multivariate analysis. $B M C$ nursing, 15 (1), 42.

[36] Vaez, M., Josephson, M., Vingård, E., \& Voss, M. (2014). Work-related violence and its association with self-rated general health among public sector employees in Sweden. Work, 49 (1), 163-171.

[37] Zhang, S.-E., Liu, W., Wang, J., Shi, Y., Xie, F., Cang, S.,... Fan, L. (2018). Impact of workplace violence and compassionate behaviour in hospitals on stress, sleep quality and subjective health status among Chinese nurses: a cross-sectional survey. BMJ open, 8 (10), e019373. 